\title{
Flexible and robust patterning by centralized gene networks.
}

\author{
S. Vakulenko ${ }^{1}$ and O. Radulescu ${ }^{2}$ \\ ${ }^{3}$ Saint Petersburg State University of Technology and Design, St.Petersburg, Russia, \\ ${ }^{4}$ DIMNP UMR CNRS 5235, University of Montpellier 2, Montpellier, France.
}

July 21, 2021

\begin{abstract}
We investigate the possibility of programming arbitrarily complex space-time patterns, and transitions between such patterns, by gene networks. We consider networks with two types of nodes. The $v$-nodes, called centers, are hyperconnected and interact one to another via $u$-nodes, called satellites. This centralized architecture realizes a bow-tie scheme and possesses interesting properties. Namely, this organization creates feedback loops that are capable to generate any prescribed patterning dynamics, chaotic or periodic, or stabilize a number of prescribed equilibrium states. We show that activation or silencing of a node can sharply switch the network dynamics, even if the activated or silenced node is weakly connected. Centralized networks can keep their flexibility, and still be protected against environmental noises. Finding an optimized network that is both robust and flexible is a computationally hard problem in general, but it becomes feasible when the number of satellites is large. In theoretical biology, this class of models can be used to implement the Driesch-Wolpert program, allowing to go from morphogen gradients to multicellular organisms.
\end{abstract}

\section{Introduction}

The richness of Alan Turing's ideas hides somehow their unity. Is there a relation between the "chemical theory of morphogenesis" (Turing 1952) and the "universal machine", or other, less known works, such as "Intelligent machinery" (Turing 1969, Teuscher and Sanchez 2001) in which he anticipates random binary networks? As emphasized by M.H.A. Newman (Newman 1955), a common denominator of Turing's scientific work is the quest for a mechanical explanation of nature. However, an even deeper unifying idea concerns the computability of nature and reciprocally, how nature computes. Turing looked for a mechanical support for natural pattern computation and found an analog machine, working by the chemical morphogens. Had he had known about gene networks, he would have probably analyzed the computational capacity of these networks to make patterns and multicellular organisms. In this paper we discuss a particular class of gene networks, the centralized or bow tie networks, and show that they can "compute" multicellular organisms and comply with important desiderata of life such as flexibility and robustness.

Flexibility and robustness are important properties of living systems in general, most particularly observed during development from egg or embryo to a large, fully organized organism. Flexibility means the capacity to change, when environmental conditions vary. Opposite to this, robustness is the capacity to support homeostasis in spite of external changes. Intriguingly, biological systems are in the same time robust and flexible. Organization of the body plan in development, should be robust under unavoidable fluctuations of maternal gradients, embryo size, and environment conditions (for instance, temperature). 
This is a viability condition. On the other hand, developmental systems should be flexible in order to produce a number of different patterns and complicated dynamics.

Pattern formation processes are important for the body plan establishment via cell fate decisions in multicellular organisms. Mathematical modeling of these phenomena (Turing 1952, Meinhardt 1982, Murray 1993, Wolpert et al. 2002, Mjolness et al. 1991, Reinitz and Sharp 1995, Page et al. 2005) is based on systems of reaction-diffusion equations, sometimes with spatial inhomogeneous reaction terms. Two different approaches, both considering that laws of physics and chemistry are sufficient to account for making of a multicellular organism, are fundamental in modeling of body plan organization. The first approach, pioneered by the seminal work of Turing (1952), uses diffusion-driven instability as a patterning mechanism. For Turing's mechanism, a spatial dependence of the reaction term is not necessary, and the translation symmetry breaking needed for body plan organization results from the Turing instability. The second approach is based on Driesch-Wolpert positional information (Wolpert et al. 2002, Wolpert 1970). The corresponding models can be also based on reaction-diffusion equations, but in this case the models have space dependent reaction terms and no translation symmetry, therefore Turing instability is not needed. The main example of this type of models is the gene circuit model (Mjolness et al 1991, Reinitz and Sharp 1995). In this case, spatial organization is triggered by pre-patterns of signaling molecules, generically called morphogen gradients. It is remarkable that germs of this second approach can be found in the conclusion of Turing's 1952 paper, where it is suggested that "most of a organism, most of the time, is developing from one pattern into another, rather than from homogeneity into a pattern".

The body plan, considered as well defined, stable sequence of transitions from one pattern to another, can be encoded in a system of gene-gene interactions or gene network. For instance, in the segmentation along the anterior-posterior axis of Drosophila (fruit-fly) embryos, the chemical support of the pre-pattern is the maternal bicoid gradient developed in eggs soon after fecundation. This gradient induces spatially localized expression of segmentation genes (hunchback, krüppel, giant, knirps, tailless, fushi tarazu, even skipped, runt, hairy, odd skipped, paired, sloppy paired, etc.) forming a gene network. This gene network employs several types of interactions to stabilize the segmentation pattern. Some of these interactions originate in trans, i.e., far, on the DNA sequence, from the gene, and are due to transcription factors (TF) and microRNAs (miRNA). Other interactions originate in cis, i.e., close, on the DNA sequence, to the gene. Indeed, the zygotic genome contains cis-regulatory elements (CREM) controlling expression of the segmentation genes. The gene circuit model (Mjolness et al 1991, Reinitz and Sharp 1995) accounts for part of the trans interactions, considering that segmentation genes are mutually regulated transcription factors. The miRNA and the CREMs interactions are not represented in this model. Although the role in stabilizing the development has been experimentally proven for miRNAs(Li et al. 2009) and for CREMs (Ludwig et al. 2011), the mechanistic details of these interactions are still unknown. MiRNAs and CREMs can be abstractly considered as intermediate nodes in a gene network, mediating interactions between transcription factors. In such networks, TFs can be target hubs, being controlled by many CREMs, and also source hubs, because they can bind to many CREMs. Similarly, a few bioinformatics studies (Shalgi et al. 2007) suggest the existence of many genes submitted to extensive miRNA regulation with many TF among these target hubs. Direct testing of these interactions have recently shown that important transcription factors can be regulated by multiple miRNA ( $\mathrm{Tu}$ and Bassler 2006, Martinez et al 2008, $\mathrm{Wu}$ et al 2010, Peter 2010). Without excluding other applications of our mathematical framework, we consider the TF-miRNAs networks as well as the TF-CREMs networks as possible examples of centralized networks, or bow-tie networks.

The main goal of this paper is to show, by rigorous mathematical methods, the following new results:

i centralized networks can create "a multicellular organism" consisting of many specialized cells where the network dynamics within each cell can have a different attractor,

ii this pattern is robust under variations of morphogenetic fields; our system performs trade-offs between flexibility and robustness,

iii bifurcations between attractors can be obtained by gene silencing or reactivation.

These results, however, would be useless, without algorithms that can resolve, in polynomial time 
$\operatorname{Poly}(N)$, (where $N$ is the gene number), the problem of a prescribed complicated and robust pattern construction ("computation of a robust organism", CRO problem). It is one of the key questions in development, why evolution had a sufficient time to construct complicated organs and organisms (Darwin, Origins of Species, Chapter 6). This problem is, in fact, a hard combinatorial one. Using new ideas in such problems, we show, under some assumptions, that

iv for centralized networks with large hub connectivity, the CRO problem is feasible in polynomial $\operatorname{Poly}(N)$ time.

Similar ideas, that bow-tie connectivity can play a role in flexibility and robustness, have been proposed by (Csete and Doyle 2004, Ma et al. 2007) in the context of metabolism, but lacked mathematical proofs. In theoretical computer science, it was shown that artificial neural networks can simulate any Turing machine (Siegelmann and Sontag 1991, 1995). Also, it was shown that networks can simulate any time trajectories (Funahashi and Nakamura 1993) and any attractors (Vakulenko 1994, 2000, Vakulenko and Gordon 1998).

We extend these results to simulation of any spatio-temporal structure, with any attractors. Since the pioneering ideas of Delbrück (1949), it became well accepted that differentiation and specialization of initially undifferentiated clone cells can be understood via multiple dynamical structures and attractors (Thomas 1998). In particular, differences between gene expression programs can be understood as differences between attractors of dynamical gene networks. At least mathematically, the possibility to control any spatio-temporal pattern is equivalent to the possibility to organize any multicellular organism. Thus, we show that centralized networks can be used to implement Driesch-Wolpert positional information paradigm in order to organize a multicellular organism. This organism consists of a number of specialized cells, each cell type being dynamically characterized by distinct attractors. The complexity of the attractors, that can be arbitrarily large, can be programmed by gradients of morphogens. Transitions between attractors can be performed by acting on key nodes of the network. Contrary to previous theories of random networks (Kauffman 1969, Aldana 2003, Aldana and Cluzel 2003), these key nodes do not have to be hubs. Furthermore, we show that patterning in such networks is maximally flexible in the sense that it can produce any structurally stable attractor. We also prove that (and show how) optimally flexible and robust structures can be computed in polynomial time and can thus be easily attained by evolution.

The paper is organized as follows. Centralized networks are introduced in Section 2. A first theorem (Proposition 2.3) concerns with the flexibility of general centralized networks. We show that these networks are capable to generate practically all structurally stable prescribed dynamics, chaotic or periodic, and can have any number of equilibrium states. Another key result (Theorem 2.5) can be interpreted, in biological terms, as follows. The centralized networks are capable to create a "multicellular organism", where each cell have a prescribed time dynamics. This assertion can be considered as a mathematical realization of the Wolpert approach since this intrinsic dynamics in a cell is predetermined only by the morphogen concentration in this cell. In Section 3 we show that gene activation or silencing can produce a sharp change of dynamics even if this gene is weakly connected in the network (it is well known that a mutation in a hub can sharply change the dynamics, see Aldana 2003). We show that in such a way one can obtain arbitrary bifurcations. In Section 4 we consider the robustness of centralized networks and show how these can acquire protection against environmental perturbations. We show that the design of a network that is both flexible and robust can be stated as an optimization problem for a discrete spin hamiltonian. When the number of satellites $N$ is large, the optimization problem can be solved in polynomial time, $\operatorname{Poly}(N)$.

\section{Centralized networks}

By centralized networks we mean networks that contain a few strongly connected nodes (hubs) and a number of less connected, satellite nodes. A typical example is given by scale-free networks (Albert and Barabasi 2002, Lesne 2006), that occur in many areas, in economics, biology and sociology. In the scalefree networks the probability $P(k)$ that a node is connected with $k$ neighbors, has the asymptotics $C k^{-\gamma}$, 
with $\gamma \in(2,3)$. Such networks typically contain a few hubs and a large number of satellite nodes. Hence, scale-free networks are, in a sense, centralized.

In order to model dynamics of centralized networks we adapt a gene circuit model proposed to describe early stages of Drosophila (fruit-fly) morphogenesis (Mjolness et al. 1991, Reinitz and Sharp 1995). To take into account the two types of the nodes, we use distinct variables $v_{j}, u_{i}$ for the centers and the satellites. The real matrix entry $A_{i j}$ defines the intensity of the action of a center node $j$ on a satellite node $i$. This action can be either a repression $A_{i j}<0$ or an activation $A_{i j}>0$. Similarly, the matrices $\mathbf{B}$ and $\mathbf{C}$ define the action of the centers on the satellites and the satellites on the centers, respectively. Let us assume that a satellite does not act directly on another satellite. We also assume that satellites respond more rapidly to perturbations and are more diffusive/mobile than the centers.

Let $M, N$ be positive integers, and let $\mathbf{A}, \mathbf{B}$ and $\mathbf{C}$ be matrices of the sizes $N \times M, M \times M$ and $M \times N$ respectively. We denote by $\mathbf{A}_{i}, \mathbf{B}_{j}$ and $\mathbf{C}_{j}$ the rows of these matrices. To simplify formulas, we use the notation

$$
\sum_{j=1}^{M} A_{i j} v_{j}=\mathbf{A}_{i} v, \quad \sum_{l=1}^{M} B_{j l} v_{l}=\mathbf{B}_{j} v, \quad \sum_{k=1}^{N} C_{j k} u_{k}=\mathbf{C}_{j} u .
$$

Then, the gene circuit model reads:

$$
\begin{gathered}
\frac{\partial u_{i}}{\partial t}=\tilde{d}_{i} \Delta u_{i}+\tilde{r}_{i} \sigma\left(\mathbf{A}_{i} v+\tilde{b}_{i} m(x)-\tilde{h}_{i}\right)-\tilde{\lambda}_{i} u_{i}, \\
\frac{\partial v_{j}}{\partial t}=d_{j} \Delta v_{j}+r_{j} \sigma\left(\mathbf{B}_{j} v+\mathbf{C}_{j} u+b_{j} m(x)-h_{j}\right)-\lambda_{j} v_{j},
\end{gathered}
$$

where $m(x)$ represents the maternal morphogen gradient, $i=1, \ldots, N, j=1, \ldots, M$. We assume that the diffusion coefficient $d_{i}, \tilde{d}_{i}$ and maximal production rates $r_{i}, \tilde{r}_{i}$ are non-negative: $d_{i}, \tilde{d}_{i}, r_{i}, \tilde{r}_{i} \geq 0$. Here the morphogenetic field $m(x)$ and unknown gene concentrations $u_{i}(x, t), v_{j}(x, t)$ are defined in a compact domain $x \in \Omega(\operatorname{dim}(\Omega) \leq 3)$ having smooth boundary $\partial \Omega, x \in \Omega$ and $\sigma$ is a monotone and smooth (at least twice differentiable) "sigmoidal" function such that

$$
\sigma(-\infty)=0, \quad \sigma(+\infty)=1 .
$$

Typical examples can be given by

$$
\sigma(h)=\frac{1}{1+\exp (-h)}, \quad \sigma(h)=\frac{1}{2}\left(\frac{h}{\sqrt{1+h^{2}}}+1\right) .
$$

The function $\sigma(\beta x)$ becomes a step-like function as its sharpness $\beta$ tends to $\infty$.

We also set the Neumann boundary conditions

$$
\nabla u_{i}(x, t) \cdot \mathbf{n}(x)=0, \quad \nabla v_{j}(x, t) \cdot \mathbf{n}(x)=0, \quad(x \in \partial \Omega) .
$$

They mean that the flux of each reagent through the boundary is zero (here $\mathbf{n}$ denotes the unit normal vector towards the boundary $\partial \Omega$ at the point $x$ ). Moreover, we set the initial conditions

$$
u_{i}(x, 0)=\tilde{\phi}_{i}(x) \geq 0, \quad v_{j}(x, 0)=\phi_{j}(x) \geq 0 \quad(x \in \Omega) .
$$

It is natural to assume that all concentrations are non-negative at the initial point, and it is easy to show that they stay non-negative for all times (see below).

Neglecting diffusion effects we obtain from (12),(2) the following shorted system:

$$
\frac{\partial u_{i}}{\partial t}=\tilde{r}_{i} \sigma\left(\mathbf{A}_{i} v+\tilde{b}_{i} m(x)-\tilde{h}_{i}\right)-\tilde{\lambda}_{i} u_{i},
$$




$$
\frac{\partial v_{j}}{\partial t}=r_{j} \sigma\left(\mathbf{B}_{j} v+\mathbf{C}_{j} u+b_{j} m(x)-h_{j}\right)-\lambda_{j} v_{j}
$$

This is a Hopfield-like network model (Hopfield 1982) with thresholds depending on $x$ (contrary to the Hopfield model, the interaction matrices are not necessarily symmetric). In this case we remove all boundary conditions (5). If only $d_{i}=0$ we remove the corresponding boundary conditions for $v_{i}$.

\subsection{Existence of solutions}

Let us introduce some special functional spaces (Henry, 1981). Let us denote $H=L_{2}(\Omega)^{n}$ the Hilbert space of the vector value functions $w$. This space is enabled by the standard $L_{2^{-}}$norm defined by $\|w\|^{2}=\int_{\Omega}|w(x)|^{2} d x$, where $|w|^{2}=\sum w_{i}^{2}$. For $\alpha>0$ we denote by $H_{\alpha}$ the space consisting of all functions $w \in H$ such that the norm $\|w\|_{\alpha}$ is bounded, here $\|w\|_{\alpha}^{2}=\left\|(-\Delta+I)^{\alpha} w\right\|^{2}$. These spaces have been well studied (see Henry 1981 and references therein). The phase space of our system is $\mathcal{H}=\{w=$ $(u, v): u \in H, v \in H\}$, the corresponding natural fractional spaces are denoted by $H_{\alpha}$ and $\mathcal{H}_{\alpha}$, here $H_{0}=H$ and $\mathcal{H}_{0}=\mathcal{H}$. Denote by $B_{\alpha}(R)$ the $n$-dimensional ball in $H_{\alpha}$ centered at the origin with the radius $R: B_{\alpha}(R)=\left\{w: w \in H_{\alpha},\|w\|_{\alpha}<R\right\}$.

In our case all $f_{i}(w, x)$ are smooth in $w, x$, therefore, the standard technique (Henry 1981) shows that solutions of (11), (2) exist locally in time and are unique. In fact, our system can be rewritten as an evolution equation of the form

$$
w_{t}=A w+f(w),
$$

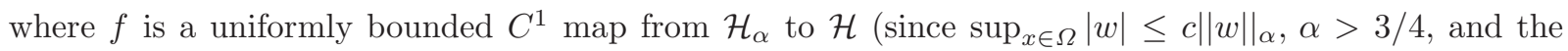
derivative $\sigma^{\prime}(z)$ is uniformly bounded in $z$ ) and a linear self-adjoint negatively defined operator $A$ generates a semigroup satisfying the estimate $\|\exp (A t) w\| \leq \exp (-\beta t)\|w\|$ with a $\beta>0$.

Let us prove that the gene network dynamics is correctly defined for all $t$ and solutions are non-negative and bounded. In fact, there exists an absorbing set $\mathcal{B}$ defined by

$$
\mathcal{B}=\left\{w=(u, v): 0 \leq v_{j} \leq r_{j} \lambda_{j}^{-1}, 0 \leq u_{i} \leq \tilde{r}_{i} \tilde{\lambda}_{i}^{-1}, j=1, \ldots, M, i=1, \ldots, N\right\} .
$$

One can show, by super and subsolutions, that

$$
\begin{aligned}
& 0 \leq u_{i}(x, t) \leq \tilde{\phi}_{i}(x) \exp \left(-\tilde{\lambda}_{i} t\right)+\tilde{r}_{i} \tilde{\lambda}_{i}^{-1}\left(1-\exp \left(-\tilde{\lambda}_{i} t\right)\right), \\
& 0 \leq v_{i}(x, t) \leq \phi_{i}(x) \exp \left(-\lambda_{i} t\right)+r_{i} \lambda_{i}^{-1}\left(1-\exp \left(-\lambda_{i} t\right)\right) .
\end{aligned}
$$

Therefore, solutions of (11), (2) not only exist for all times $t$ but also they enter the set $\mathcal{B}$ at a time moment $t_{0}$ and then they stay in this set for all $t>t_{0}$. So, our system defines a global dissipative semiflow (Henry, 1981).

\subsection{Reduced dynamics}

The key idea is to find a simpler asymptotic description of system dynamics. It is possible under some assumptions, we suppose here that the $u$-variables are fast and the $v$-ones are slow. We show then that the fast $u$ variables are captured, for large times, by the slow $v$ modes. More precisely, one has $u=U(v)+\tilde{u}$, where $\tilde{u}$ is a small correction. This means that, for large times, the satellite dynamics is defined almost completely by the center dynamics.

To realize this approach, let us assume that the parameters of the system satisfy the following conditions:

$$
\left|A_{j l}\right|,\left|B_{i l}\right|,\left|C_{i j}\right|,\left|\tilde{h}_{i}\right|,\left|h_{j}\right|<C_{0},
$$

where $i=1,2, \ldots, N, \quad i, l=1, \ldots, M, j=1, \ldots, N$,

$$
0<C_{1}<\tilde{\lambda}_{j}, \quad \tilde{d}_{j}<C_{2},
$$




$$
\left|b_{j}\right|,\left|\tilde{b}_{i}\right|<C_{3}, \quad \sup |m(x)|<C_{4},
$$

and

$$
r_{i}=\kappa R_{i}, \quad \tilde{r}_{i}=\kappa \tilde{R}_{i},
$$

where

$$
\begin{array}{cl}
\left|R_{i}\right|,\left|\tilde{R}_{i}\right|<C_{5}, & \lambda_{i}=\kappa \bar{\lambda}_{i},|\bar{\lambda}|<C_{6}, \\
d_{j}=\kappa \bar{d}_{j}, & 0<\bar{d}_{j}<C_{7},
\end{array}
$$

where $\kappa$ is a small parameter, and where all positive constants $C_{k}$ are independent of $\kappa$.

Proposition 2.1. Assume the space dimension Dim $\Omega \leq 3$. Under assumptions (11), (12), (13), (14) for sufficiently small $\kappa<\kappa_{0}$ solutions $(u, v)$ of (1), (2), (5), and (6) satisfy

$$
u=U(x, v(x, t))+\tilde{u}(x, t),
$$

where the $j$-th component $U_{j}$ of $U$ is defined as a unique solution of the equation

$$
\tilde{d}_{j} \Delta U_{j}-\tilde{\lambda}_{j} U_{j}=\kappa G_{j}(v),
$$

under the boundary conditions (5), where

$$
G_{j}=\tilde{R}_{j} \sigma\left(\mathbf{A}_{j} v(x, t)+\tilde{b}_{j} m(x)-\tilde{h}_{j}\right)
$$

The function $\tilde{u}$ satisfies the estimates

$$
\|\tilde{u}\|+\|\nabla \tilde{u}\|<c_{1} \kappa^{2}+R \exp (-\beta t), \quad \beta>0 .
$$

The $v$ dynamics for large times $t>C_{1}|\log \kappa|$ takes the form

$$
\frac{\partial v_{i}}{\partial t}=\kappa F_{i}(u, v)+w_{i}
$$

where $w_{i}$ satisfy

$$
\left\|w_{i}\right\|<c_{0} \kappa^{2}
$$

and

$$
F_{i}(u, v)=\bar{d}_{i} \Delta v_{i}+R_{i} \sigma\left(\mathbf{B}_{i} v+\mathbf{C}_{i} U(x, v)+b_{i} m-h_{i}\right)-\bar{\lambda}_{i} v_{i} .
$$

Constants $c_{0}, c_{1}$ do not depend on $\kappa$ as $\kappa \rightarrow 0$ but they may depend on $R_{i}, \tilde{R}_{i}, C_{i}$.

A tedious proof of this assertion is basically straightforward; it is based on well known results (Henry 1981) and is relegated to the Appendix.

An analogous assertion holds for shorted system (7), (8). In this case the functions $U_{i}$ can be found by an explicit formula. Namely, one has

$$
U_{i}(x, v(x, t))=\kappa V_{i}, \quad V_{i}=R_{i} \tilde{\lambda}_{i}^{-1} \sigma\left(\mathbf{A}_{j} v(x, t)+\tilde{b}_{j} m(x)-\tilde{h}_{j}\right) .
$$

For large times the reduced $v$ dynamics has the same form (20) with $d_{i}=0$. 


\subsection{Realization of prescribed dynamics by networks}

Our next goal is to show that dynamics (20) can realize, in a sense, arbitrary structurally stable dynamics of the centers. To precise this, let us describe the method of realization of the vector fields for dissipative systems (proposed by Poláčik (1991), for applications see, for example, (Dancer and Poláčik 1999, Rybakowski 1994, Vakulenko 2000). One can show that some systems possess the following properties:

A These systems generate global semiflows $S_{\mathcal{P}}^{t}$ in an ambient Hilbert or Banach phase space $H$. These semiflows depend on some parameters $\mathcal{P}$ (which could be elements of another Banach space $\mathcal{B}$ ). They have global attractors and finite dimensional local attracting invariant $C^{1}$ - manifolds $\mathcal{M}$, at least for some $\mathcal{P}$.

(Remark: in some cases, these manifolds can be even globally attracting, i.e., inertial. Theory of invariant and inertial manifold is well developed, see (Marion 1989, Mane 1977, Constantin et al 1989, Chow and Lu 1988, Babin and Vishik 1988).

B Dynamics of $S_{\mathcal{P}}^{t}$ reduced on these invariant manifolds is, in a sense, "almost completely controllable". It can be described as follows. Assume the differential equations

$$
\frac{d p}{d t}=F(p), \quad F \in C^{1}\left(B^{n}\right)
$$

define a dynamical system in the unit ball $B^{n} \subset \mathbf{R}^{n}$.

For any prescribed dynamics (22) and any $\delta>0$, we can choose suitable parameters $\mathcal{P}=\mathcal{P}(n, F, \delta)$ such that

B1 The semiflow $S_{\mathcal{P}}^{t}$ has a $C^{1}$ - smooth locally attracting invariant manifold $\mathcal{M}_{\mathcal{P}}$ diffeomorphic to $B^{n}$; B2 The reduced dynamics $\left.S_{\mathcal{P}}^{t}\right|_{\mathcal{M}_{\mathcal{P}}}$ is defined by equations

$$
\frac{d p}{d t}=\tilde{F}(p, \mathcal{P}), \quad \tilde{F} \in C^{1}\left(B^{n}\right)
$$

where the estimate

$$
|F-\tilde{F}|_{C^{1}\left(B^{n}\right)}<\delta
$$

holds. In other words, one can say that, by $\mathcal{P}$, the inertial dynamics can be specified to within an arbitrarily small error.

Thus, all robust dynamics (stable under small perturbations) can occur as inertial forms of these systems. Such systems can be named maximally dynamically flexible, or, for brevity, MDF systems.

Such structurally stable dynamics can be "chaotic". There is a rather wide variation in different definitions of "chaos". In principle, one can use here any concept of chaos, provided that this is stable under small $C^{1}$-perturbations. To fix ideas, we shall use here, following classical tradition (Ruelle and Takens 1971, Newhouse, Ruelle and Takens 1971, Smale 1980, Anosov 1995), such a definition. We say that a finite dimensional dynamics is chaotic if it generates a hyperbolic invariant set $\Gamma$, which is not a periodic cycle or a rest point. For a definition of hyperbolic sets see, for example, (Ruelle 1989); a famous example is given by the Smale horseshoe. If, moreover, this set $\Gamma$ is attracting we say that $\Gamma$ is a chaotic (strange) attractor. In this paper, we use only the following basic property of hyperbolic sets, so-called Persistence (Ruelle 1989, Anosov 1995). This means that the hyperbolic sets are, in a sense, stable(robust): if (22) generates the hyperbolic set $\Gamma$ and $\delta$ is sufficiently small, then dynamics (23) also generates another hyperbolic set $\tilde{\Gamma}$. Dynamics (22) and (23) restricted to $\Gamma$ and $\tilde{\Gamma}$ respectively, are topologically orbitally equivalent (on definition of this equivalence, see Ruelle 1989, Anosov 1995).

Thus, any kind of the chaotic hyperbolic sets can occur in the dynamics of the MDF systems, for example, the Smale horseshoes, Anosov flows, the Ruelle-Takens-Newhouse chaos, see (Newhouse, Ruelle and Takens 1971, Smale 1980, Ruelle 1989). Examples of systems satisfying these properties can be given by some reaction diffusion systems (Dancer and Poláčik 1999, Rybakowski 1994, Vakulenko 2000). 
Although not yet observed in gene networks, structurally stable chaotic itineracy is thought to play a functional role in neuroscience (Rabinovitch 1998).

Let us apply this approach to network dynamics using the results of the previous section. To this end, assume that (14), 15) and (16) hold. Moreover, let us assume

$$
\begin{array}{cc}
b_{i}=\kappa \bar{b}_{i}, & h_{i}=\kappa \bar{h}_{i} \\
\lambda_{i}=\kappa^{2} \bar{\lambda}_{i}, & d_{i}=\kappa^{2} \bar{d}_{i}
\end{array}
$$

where all coefficients $\bar{b}_{i}$ and $\bar{h}_{i}$ are uniform in $\kappa$ as $\kappa \rightarrow 0$. These assumptions are useful for technical reasons. We also assume that all direct interactions between centers are absent, $\mathbf{B}=\mathbf{0}$. This constraint is not essential but facilitates notation and calculations.

Since $U_{j}=O(\kappa)$ for small $\kappa$, we can use the Taylor expansion for $\sigma$ in (20). Then these equations reduce to

$$
\frac{\partial v_{i}(x, \tau)}{\partial \tau}=\bar{d}_{i} \Delta v_{i}+\rho_{i}\left(\mathbf{C}_{i} V(x, v)+\bar{b}_{i} m(x)-\bar{h}_{i}\right)-\bar{\lambda}_{i} v_{i}+\tilde{w}_{i}(x, t),
$$

where $\rho_{i}(x)=\bar{r}_{i} \sigma^{\prime}(0), i=1,2, \ldots, M$ and $\tau$ is a slow rescaling time: $\tau=\kappa^{2} t$. Due to conditions (25) and (26) corrections $\tilde{w}_{i}$ satisfy

$$
\left\|\tilde{w}_{i}\right\|<c \kappa .
$$

Let us focus now our attention to non-perturbed equation (27) with $\tilde{w}_{i}=0$. Let us fix the number of centers $M$. The number of satellites $N$ will be considered as a parameter.

The next important lemma follows from known approximation theorems of multilayered network theory, see, for example, (Barron 1993, Funahashi and Nakamura 1993).

Lemma 2.2. Given a number $\delta>0$, an integer $M$ and a vector field $F=\left(F_{1}, \ldots, F_{M}\right)$ defined on the ball $B^{M}=\{|v| \leq 1\}, F_{i} \in C^{1}\left(B^{M}\right)$, there are a number $N$, a $N \times M$ matrix $\mathbf{A}$, a $M \times N$ matrix $\mathbf{C}$ and coefficients $h_{i}$, where $i=1,2, \ldots, N$, such that

$$
\left|F_{j}(\cdot)-\mathbf{C}_{j} W(\cdot)\right|_{C^{1}\left(B^{M}\right)}<\delta
$$

where

where $v=\left(v_{1}, \ldots, v_{M}\right) \in \mathbf{R}^{M}$.

$$
W_{i}(v)=\sigma\left(\mathbf{A}_{i} v-h_{i}\right)
$$

This lemma gives us a tool to control network dynamics and patterns. First we consider the case when the morphogens are absent. Formally, we can set $\tilde{b}_{i}=\bar{b}_{j}=\bar{d}_{i}=0$. Assume $\bar{h}_{i}=0$. Then equations (27) with $\tilde{w}_{i}=0$ reduce to the Hopfield-like equations for variables $v_{i} \equiv v_{i}(\tau)$ that depend only on $\tau$ :

$$
\frac{d v_{l}}{d \tau}=\mathbf{K}_{l} W(v)-\bar{\lambda}_{l} v_{l},
$$

where $l=1, \ldots, M$, the matrix $\mathbf{K}$ is defined by $K_{l j}=\rho_{l} C_{l j} R_{j} \tilde{\lambda}_{j}^{-1}$. The parameters $\mathcal{P}$ of (30) are $\mathbf{K}, M$, $h_{j}$ and $\bar{\lambda}_{j}$.

In this case one can formulate the following result.

Proposition 2.3. Let us consider a $C^{1}$-smooth vector field $Q(p)$ defined on a ball $B^{M} \subset \mathbf{R}^{M}$ and directed strictly inside this ball at the boundary $\partial B^{M}$ :

$$
F(p) \cdot p<0, \quad p \in \partial B^{M} .
$$

Then, for each $\delta>0$, there is a choice of parameters $\mathcal{P}$ such that (30) $\delta$-realizes the system (22). This means that (30) is a MDF system.

This proposition follows from the Prop. 2.1 and Lemma 2.2.

Prop. 2.3 implies the following important corollary: all structurally stable dynamics, including periodic and chaotic dynamics can be realized by centralized networks. The proof of this fact uses the classical results on the persistence of hyperbolic sets, and on the existence of invariant manifolds (Ruelle 1989), see (Vakulenko 2000). 


\subsection{Pattern and attractor control by Wolpert positional information}

Above we have considered a spatially homogeneous case. Proposition 2.3 shows that a centralized network can approximate an arbitrary prescribed dynamics. Thus, it is shown that cells can be programmed to have arbitrarily complex dynamics. By network rewiring or by interaction tuning, one can switch between various types of dynamics. During development these switches are position dependent, and induce cell differentiation into specific spatial arrangements.

Let us show that the centralized networks, coupled to morphogen gradients, can generate any spatiotemporal pattern as support for multicellular organization. We consider shorted dynamics (7), (8) that is reasonable for cellularized developmental stages, where cell walls prevent a free diffusion of regulatory molecules. Although other phenomena such as cell signalling can also lead to cell coupling, we do not discuss these effects here.

Assume cell positions are centered at the points $x \in \mathcal{X}=\left\{x_{1}, x_{2}, \ldots, x_{k}\right\}, \operatorname{dim} \Omega=1, \mathcal{X}$ is a discrete subset of $[0, L]$. Let us show that eqs. (77)-(8) can realize different dynamics at different points $x_{l}$ of the domain $\Omega=[0, L]$.

We can formulate now the following,

Theorem 2.5. (On translation of positional information into complex and variegated cell dynamics, or programming of multicellular organism). Suppose $x \in[0, L] \subset \mathbf{R}$ and $m(x)$ is a strictly monotone smooth function.

Assume that $0<x_{1}<x_{2}<\ldots<x_{k}<L$ and that $F^{(l)}(p), l=1,2, \ldots, k$ is a family of $C^{1}$-smooth vector fields defined on a unit ball $B^{M} \subset \mathbf{R}^{M}$. We assume that each field defines a dynamical system, i.e., $F^{(l)}$ are directed inwards on the boundary $\partial B^{M}$.

Then, for each $\delta>0$ there is a parameter $\mathcal{P}$ choice such that for shorted dynamics (7)-(8) one has

$$
u=U(x, v)+\tilde{u}
$$

where

$$
|\tilde{u}|<C \exp (-\beta \tau)+c \kappa^{2} .
$$

For $x=x_{l}$ and for sufficiently large times the dynamics for $v\left(x_{l}, t\right)$ can be reduced to the form

$$
\frac{d p_{i}}{d \tau}=\bar{F}_{i}\left(x_{l}, p\right)
$$

where

$$
\sup _{p \in B^{M}}\left|\bar{F}\left(x_{l}, p\right)-F^{(l)}(p)\right|<\delta .
$$

Here $p_{i}(\tau)$ can be expressed in a linear way via $v_{i}\left(x_{l}, \tau\right)$ by

$$
v_{i}\left(x_{l}, \tau\right)-\bar{b} m\left(x_{l}\right)=\rho_{0} p_{i}(\tau)
$$

This theorem can be considered as a mathematical formalization of positional information ideas. It extends Driesch-Wolpert theory by incorporating gene networks and coping with their information processing role. Flexible gene networks have different dynamics and attractors, for different local concentrations of morphogens. The attractor selection ensures the cell fate decision. Concerning the relation between attractors and cell fate determination, see (Delbrück 1949, Thomas 1998).

To prove this assertion, let us turn to eqs. (27), where, taking into account biological arguments given above, we set $d_{i}=0, \tilde{b}_{i}=0$. Let us set, to simplify formulas, $\rho_{j}=1, \bar{h}_{j}=0$ and $\bar{\lambda}_{j}=1$. Then

$$
V_{j}(v)=R_{j} \tilde{\lambda}_{j}^{-1} \sigma\left(\mathbf{A}_{j} v-\tilde{h}_{j}\right) .
$$


Denote by $Q_{i}$ the sums $Q_{i}(v)=\sum_{j=1}^{M} C_{i j} V_{j}(v)=\mathbf{C}_{i} V$. Removing the terms $\tilde{w}_{i}$ in (27), one obtains that eqs. (27) reduce to

$$
\frac{\partial v_{i}(x, \tau)}{\partial \tau}=Q_{i}(v(x, \tau))+\bar{b}_{i} m(x)-v_{i}(x, \tau)
$$

Let us fix a $x=x_{l} \in \mathcal{X}$. Let us make the substitution $v_{i}\left(x_{l}, \tau\right)=z_{i}(\tau)+\bar{b} m\left(x_{l}\right)$ in (34) that gives

$$
\frac{d z_{i}(\tau)}{d \tau}=Q_{i}\left(z+\bar{b} m\left(x_{l}\right)\right)-z_{i}
$$

where $\bar{b}=\left(\bar{b}_{1}, \ldots, \bar{b}_{M}\right), i=1, \ldots, M$.

Now we again use approximation Lemma 2.2. Let us consider a family of vector fields $C^{1}$-smooth vector field $F$ defined on a unit ball $B^{M}=\left\{z \in \mathbf{R}^{M},|z| \leq 1\right\}$ and directed strictly inside this ball at the boundary $\partial B^{M}$ :

$$
F^{(l)}(z) \cdot z<0, \quad z \in \partial B^{M} .
$$

Assume $m(x)$ is a strictly monotone function in $x$. The main idea is as follows: since all $m\left(x_{l}\right)=\mu_{l}$ and $m\left(x_{j}\right)=\mu_{j}$ are different for $j \neq l$, the vector fields $Q^{(l)}(z)=Q\left(z+\bar{b} \mu_{l}\right)$ can approximate different vector fields $F^{(l)}(z)$ for $l=1, \ldots, k$ and for $z$ such that $|z|<\rho_{0}$, where $\rho_{0}=\frac{1}{2} \min _{i, j, l, j \neq l}\left|\bar{b}_{i}\right|\left|\mu\left(x_{j}\right)-\mu\left(x_{l}\right)\right|$.

For each $\epsilon>0$ we can find an approximation $Q$ satisfying

$$
\left.\sup _{|z|<\rho_{0}} \mid Q\left(z+\bar{b} m\left(x_{l}\right)\right)-\left(\rho_{0} F^{(l)} \rho_{0}^{-1} z\right)+z\right) \mid<\rho_{0} \epsilon,
$$

and

$$
\left.\sup _{|z|<\rho_{0}} \mid \nabla\left(Q\left(z+\bar{b} m\left(x_{l}\right)\right)\right)-\nabla \rho_{0} F^{(l)}\left(\rho_{0}^{-1} z\right)+z\right) \mid<\rho_{0} \epsilon .
$$

Then equation (35) reduces to

$$
\frac{d z}{d \tau}=\rho_{0} F^{(l)}\left(\rho_{0}^{-1} z\right)+\rho_{0} \epsilon \tilde{F}^{(l)}(z)
$$

where

$$
\sup _{z \in B^{M}}\left|\tilde{F}^{(l)}(z)\right|<1, \quad \sup _{z \in B^{M}}\left|\nabla \tilde{F}^{(l)}(z)\right|<1 .
$$

We set $z_{i}=\rho_{0} p_{i}$. This gives

$$
\frac{d p}{d \tau}=F^{(l)}(p)+\epsilon \tilde{F}^{(l)}(p) .
$$

Let us notice that, if $\epsilon$ is small enough, then for each index $l$, due to assumption (36), the trajectory $p(t, p(0))$ stays in $B^{M}$ when the starting point lies in $B^{M}: p(0) \in B^{M}$. Consequently, our approximations (37) give vector fields that, by (39), realize different dynamics for each $x_{l}$.

\section{Sharp genetic switch by satellite silencing/reactivation}

In the context of scale-free random networks, it was proposed (Aldana 2003) that removing of a strong connected center can sharply change the network attractor. Here we will show that one can obtain transitions between all possible structurally stable attractors by a single event acting on a specially chosen weakly connected satellite. Such a satellite interacts only to one or two centers. Such event may be, either deletion, silencing, or reactivation. Therefore, such a node can serve as a switch between two kinds of network behavior. Each of the type of behavior can be defined, for example, by an attractor or several coexisting attractors that can be fixed points, periodic or chaotic attractors. 
To formalize these ideas mathematically, let us consider a system of ordinary differential equations

$$
\frac{d p}{d t}=F(p, s), \quad p \in B^{n} \subset \mathbf{R}^{n}
$$

depending on a real parameter $s$. Here $p=\left(p_{1}, p_{2}, \ldots, p_{n}\right), B^{n}$ is the unit ball centered at $p=0$, and $F$ is $C^{1}$-smooth vector field directed inside the ball at the ball boundary for each $s$ (see (36)). Let us consider $s_{0}, s_{1}$ such that $s_{0} \neq s_{1}$ and suppose that (40) has different attractors $\mathcal{A}_{0}$ and $\mathcal{A}_{1}$ for $s=s_{0}, s=s_{1}$ respectively.

Consider, for simplicity, the gene circuit model (1), (2) without diffusion and space variables:

$$
\begin{aligned}
& \frac{d u_{i}}{d t}=\tilde{r}_{i} \sigma\left(\mathbf{A}_{i} v-\tilde{h}_{i}\right)-\tilde{\lambda}_{i} u_{i}, \\
& \frac{d v_{j}}{d t}=r_{j} \sigma\left(\mathbf{C}_{j} u-h_{j}\right)-\lambda_{j} v_{j},
\end{aligned}
$$

where $i=1, \ldots, M+1, j=1, \ldots, N$. The parameters $\mathcal{P}$ of this system are $M, N, h_{i}, \tilde{h}_{i}, \lambda_{i}, r_{i}, \tilde{r}_{j}, \tilde{\lambda}_{j}$ and the matrices $\mathbf{A}, \mathbf{C}$. We can assume, without loss of generality, that we eliminate (by silencing) the $M+1$-th satellite node, $i=M+1$. As a result of this elimination, we obtain a similar system with $i=1, \ldots, M$ and shorted matrices $\mathbf{A}, \mathbf{C}$. Of course, we can also consider the opposite event, which is to reactivate the $M+1$-th node and recover the initial system this way.

Theorem 3.1 For each $\epsilon>0$ there is a choice of the parameters $\mathcal{P}$ such that system 411 , 42) with $M$ satellite nodes $\epsilon$-realizes (40) with $s=s_{0}$ and system (41), (42) with $M+1$ satellite nodes $\epsilon$-realizes (40) with $s=s_{1}$.

To prove it, we use the following extended system

$$
\begin{gathered}
\frac{d p}{d t}=\rho F(p, s), \quad p \in B^{n} \\
\frac{d s}{d t}=f(s, \beta)-\nu s, \quad s \in \mathbf{R}
\end{gathered}
$$

where $\nu>1$ and $f(s)$ is a smooth function, $\beta, \rho>0$ are parameters. Then equilibrium points $s_{e q}$ of (44) are solutions of

$$
f(s, \beta)=\nu s
$$

The point $s_{e q}$ is a local attractor if $f_{s}^{\prime}\left(s_{e q}\right)<\nu$. Let us denote $s_{e q}\left(\beta_{k}\right)=s_{k}$, where $k=0,1$, and let these roots of (45) be stable, i.e., $f^{\prime}\left(s_{k}\right)<\nu$.

Then, if $\rho>0$ is small enough, and $s_{k}$ is a single stable rest point, the fast variable $s$ approaches at $s_{e q}(\beta)$ and for large times $t$ the dynamics of our system (43), (44) is defined by the reduced equations

$$
\frac{d p}{d t}=\rho F\left(p, s_{e q}(\beta)\right)
$$

Now let us set

$$
f(s, \beta)=2 \beta^{2} \sigma\left(b\left(s-h_{0}\right)\right), \quad h_{0}<0
$$

where $b$ is a large parameter. Then $f$ is close to a step function with the step $2 \beta^{2}$. Therefore for $s_{e q}(\beta)$ one has the asymptotics $s_{e q}=2 \beta^{2} \nu^{-1}+O(\exp (-b))$ as $b \rightarrow \infty$. Thus, we can adjust parameters $\beta, b>0$ in such a way that (45) has a single stable root $s_{0}$ and the equation

$$
f(s, \beta / \sqrt{2})=\nu s
$$

also has a single root $s_{1}=\beta^{2} / \nu \neq s_{0}$. 
Dynamics (43), (44) with $f$ from (47) can be realized by a network (41), (42) in such a way. We decompose all satellites $u_{i}$ into two subsets. The first set contains satellites $u_{1}, u_{2}, \ldots, u_{M-1}$, the second one consists of the satellites $u_{M}, u_{M+1}$ ( to single out this variables, let us denote $u_{M}=y_{1}, u_{M+1}=y_{2}$ ). The main idea of this decomposition is as follows. We can linearize equations for the centers $v_{j}$ assuming that the matrix $\mathbf{C}$ is small and $\mathbf{B}=0$ (as above in Section 2).

The $y$ satellites realizes the dynamics (44) by a center $s$ :

$$
\begin{gathered}
\frac{d s}{d t}=-\nu s+\beta\left(y_{1}+y_{2}\right), \\
\frac{d y_{k}}{d t}=-y_{k}+\beta \sigma\left(b\left(s-h_{0}\right)\right), \quad k=1,2 .
\end{gathered}
$$

Here we assume that $\nu, \beta$ is small enough, therefore, for large times this system reduces to (44) with $f$ defined by (47). We see that this dynamics bifurcates into (44) with $f=\beta^{2} \sigma\left(b\left(s-h_{0}\right)\right)$ if we remove $y_{2}$ in the right hand side of (49).

The rest of the equations, after a notation modification and linearization, take the following form

$$
\begin{gathered}
\frac{d u_{i}}{d t}=\tilde{r}_{i} \sigma\left(\mathbf{A}_{i} v+D_{i} s-\tilde{h}_{i}\right)-\tilde{\lambda}_{i} u_{i}, \quad i=1, \ldots, M-1 \\
\frac{d v_{j}}{d t}=-\lambda_{j} v_{j}+r_{j} \mathbf{C}_{j} u-h_{j},
\end{gathered}
$$

where $i=1, \ldots, M+1, j=1, \ldots, N$.

Equations (51), (52) can $\epsilon$-realize arbitrary systems (40) with the parameter $s$ which can be shown as above (see Section 2), and this completes the proof.

\section{Robust dynamics}

Our definition of robust dynamics is inspired from similar ideas in viability theory (Aubin et al. 2005). Let us suppose that the dynamics (the global semiflow $S^{t}$ ), generates a number of attractors. Each attractor $\mathcal{A}$ has an attraction basin $B(\mathcal{A})$, that is an open set in the phase space. Assume that our initial data $\phi$ lie in an attractor, $\phi \in \mathcal{A}$, and let us add some noise $\xi$ to the dynamics, representing the effect of the environment. Trajectories become random, and then it is possible that, under this noise, the trajectory leaves $B(\mathcal{A})$.

We can now define the following characteristic of stability under the noise. Let us denote $P(T, B(\mathcal{A}), \phi)$ the probability that the trajectory $u(t, \phi)$ such that $u(0)=\phi \in \mathcal{A}$ stays in $B(\mathcal{A})$ within the time interval $[0, T]$.

Definition. Let us consider a network dynamics depending on some parameters $\mathcal{P}$ and on the noise $\xi$. We say that the dynamics is robust under the noise $\xi$, if for each $T$ and $\delta>0$ there is a choice of the parameters such that

$$
P(T, B(\mathcal{A}), \phi)<\delta
$$

for each attractor $\mathcal{A}$ and $\phi \in \mathcal{A}$.

\subsection{Centralized motif with noise}

For the rest of this section we consider a simplified network, with a single central node interacting with many satellites. This motif can appear as a subnetwork in a larger centralized network. In order to study robustness, we consider the case when the satellites and the center are under the influence of noise. More general situations, including perturbations of several centers and satellites, will be studied elsewhere. 
The network dynamics is described by the following equations:

$$
\begin{gathered}
\frac{\partial u_{i}}{\partial t}=d_{i} \Delta u_{i}-\lambda_{i} u_{i}+\sigma\left(b_{i} v-h_{i}+\xi_{i}(x, t)\right), \quad i=1, \ldots, N, \\
\frac{\partial v}{\partial t}=d_{0} \Delta v-\lambda_{0} v+\sigma\left(\sum_{i=1}^{N} a_{i} u_{i}-h_{0}+\xi_{0}(x, t)\right),
\end{gathered}
$$

The random fields $\xi_{i}(x, t)$ summarize the effect of various extrinsic noise sources. These can be random variations of the morphogen, or environment noise, or genetic variability affecting network interactions.

Intrinsic noise, resulting from stochastic gene expression, could be represented as supplementary terms outside the sigmoid function. In order to avoid further some tedious technical difficulties, we postpone the discussion of intrinsic noise to future work. Some aspects of the robustness of patterns with respect to intrinsic noise was studied numerically by Scott et al. (2010).

The flexibility of such simple networks results from the preceding section.

We can formulate the following problem: how to choose a network motif, robust under a given environmental noise, and simultaneously flexible? The choice can result either from genetic changes (for instance mutations, deletions or duplications of DNA regions) or from network plasticity (epigenetic changes, such as methylation and chromatin remodeling).

Assume that the considered process is a choice of satellites $u_{i}$ from a large pool of possible regulators. We can present this process as a choice of $n$ indices $j_{i}, i=1, \ldots, n$ from a larger set $I_{N}=\{1,2, \ldots, N\}$ of indices, where $N \geq n$. This choice can be done by boolean variables $s_{j}$ that multiply the coefficients $a_{j}$ : the $j$-th reagent participates in the network if $s_{j}=1$ and does not participate if $s_{i}=0$. Let us make an important assumption allowing us to obtain a thermodynamical limit as $N \rightarrow \infty$. We assume that

$$
\left|a_{i}\right|<C N^{-1}, \quad N \rightarrow \infty .
$$

Now we transform eqs. (53), (54), using the results of the previous subsection. Let $v=q(x)$ and $u_{i}=U_{i}(x)$ be equilibrium solutions of (53), (54) where the noises $\xi_{i}$ are removed. We suppose that the assumptions of the previous subsection hold. Let us set

$$
v=q+\tilde{v}, \quad u_{i}=U_{i}+\tilde{u}_{i}
$$

and $U, u$ denote vectors $\left(U_{1}, \ldots, U_{N}\right),\left(u_{1}, \ldots, u_{N}\right)$. Let us set temporarily $\xi_{0}=0$ ( below we shall show how one can stabilize the system state, when $\left.\xi_{0} \neq 0\right)$. This gives

$$
\frac{\partial \tilde{v}}{\partial t}=d_{0} \Delta \tilde{v}-\lambda_{0} \tilde{v}+\sigma(\rho(U+\tilde{u})-\bar{h})-\sigma(\rho(U)-\bar{h})
$$

where we use, for brevity, the notation $\rho(u)=\sum_{i=1}^{N} s_{i} a_{i} u_{i}$.

The second part of equations takes then the form

$$
\frac{\partial \tilde{u}_{i}}{\partial t}=-d_{i} \Delta \tilde{u}_{i}-\lambda_{i} \tilde{u}_{i}+\sigma\left(b_{i}(q+\tilde{v})+\xi_{i}-h_{i}\right)-\sigma\left(b_{i} q-h_{i}\right) .
$$

To investigate equations (56), (57), we use a special method justified in a rigorous way in Appendix. This holds under the following assumption:

Assumption 4.4. The "morphogenetic" noises $\xi_{i}(x, t)$ are independent on $t$ :

$$
\xi_{i}(x, t)=\xi_{i}(x), \quad i=0,1 \ldots, N
$$

The functions $\xi_{i}$ are continuous in $x$ and a priori bounded

$$
\sup _{x \in \Omega}\left|\xi_{i}(x)\right|<C_{*}, \quad i=0,1, \ldots, N .
$$


where a positive constant $C_{*}$ may be large but it is independent of $N$ for large $N$.

Notice that Assumption 4.4 guarantees global existence of solutions $\tilde{u}_{i}(x, t), \tilde{v}(x, t)$ of eqs. (56), (57) for all $t>0$.

Intuitively, one can expect that the term $\tilde{v}$ in (57) in $\sigma$ is small and can be, thus, removed. Following this idea, let us introduce $\eta_{i}$ as solutions of

$$
\frac{\partial \eta_{i}}{\partial t}=-d_{i} \Delta \eta_{i}-\lambda_{i} \eta_{i}+\sigma\left(b_{i} q+\xi_{i}-h_{i}\right)-\sigma\left(b_{i} q-h_{i}\right) .
$$

If $\xi_{i}$ are independent of $t$, and sufficiently regular in $x$ then arguments of the previous section show that for large $t$

$$
\eta_{i}(x, t) \rightarrow \bar{\eta}_{i}(x)
$$

where $\bar{\eta}_{i}$ are solutions of elliptic equations

$$
d_{i} \Delta \bar{\eta}_{i}+\lambda_{i} \bar{\eta}_{i}=G_{i}\left(\xi_{i}(x)\right), \quad G_{i}\left(\xi_{i}(x)\right)=\sigma\left(b_{i} q+\xi_{i}-h_{i}\right)-\sigma\left(b_{i} q-h_{i}\right)
$$

under zero Neumann boundary conditions.

Let us consider equation (56) for $v$. Assume $\rho$ is small. Then we can linearize the nonlinear contributions in the right hand side of this equation:

$$
\sigma(\rho(U+\tilde{u})-\bar{h})-\sigma(\rho(U)-\bar{h})=\sigma^{\prime}(U-\bar{h}) \rho(\tilde{u})+O\left(\rho(\tilde{u})^{2}\right) .
$$

We assume that $\tilde{u}_{i}$ are close to $\bar{\eta}_{i}$. Thus, $\rho(\tilde{u}) \approx \rho(\bar{\eta})$. Calculations presented in the Appendix show that the fluctuation influence can be estimated through the quantity

$$
\delta(s, T)=\sup _{t \in[0, T]} \mathbf{H}(s, t), \quad \mathbf{H}(s, t)=\|\rho(\bar{\eta})\|^{2} .
$$

If $\xi_{i}$ are independent of $t$, for large $t$ one has

$$
\mathbf{H}(s, t) \rightarrow \overline{\mathbf{H}}(s)=\|\rho(\bar{\eta})\|^{2} .
$$

Notice that $\overline{\mathbf{H}}$ can be rewritten in the form

$$
\overline{\mathbf{H}}(s)=\sum_{m=1}^{N} \sum_{m^{\prime}=1}^{N} W_{m m^{\prime}}(\bar{\eta}(\cdot)) s_{m} s_{m^{\prime}},
$$

where $W_{m m}$ are random and

$$
W_{m m^{\prime}}(\bar{\eta}(\cdot))=a_{m} a_{m^{\prime}}\left\langle\bar{\eta}_{m}, \bar{\eta}_{m^{\prime}}\right\rangle,
$$

here $\langle f, g\rangle$ denotes the inner scalar product in $H:\langle f, g\rangle=\int_{\Omega} f g d x$, where $d x$ is the standard Lebesgue measure.

\subsection{Hard combinatorial problems in network evolution}

We assume that Assumption 4.4 holds. The minimization of $\mathbf{H}(s)$ with respect to $s$ should be done under the condition that at least one satellite is involved, i.e.,

$$
R_{0}(s)=N^{-1} \sum_{i=1}^{N} s_{i}>0 .
$$

The analysis of the minimization problem for this random Hamiltonian is a computationally hard problem advanced firstly by methods from statistical physics of spin glasses (see, for example, (Mezard Zecchina 
2002) for applications to hard combinatorial problems, and (Talagrand 2003) for rigorous justification). To make the analogy with spin glasses more transparent, we can make change $s_{i}=2 S_{i}+1$, where spin variables $S_{i}$ take values 1 or -1 . However, our problem is even more complicated because, besides (66), some other restrictions should be taken into account.

In addition to (66), we must take into account restrictions connected with generation of several steady states $q_{1}, q_{2}, \ldots, q_{M}$, to provide flexibility. Let us take a small $\epsilon>0$. By adjusting $s_{i}$ we would like to obtain a set of equilibria close to $q_{l}$. This gives the following restrictions

$$
\sup _{x \in \Omega} \sigma\left(\sum_{i=1}^{N} s_{i} a_{i} \sigma\left(b_{i} q_{l}-\bar{h}\right)-\lambda_{0} q_{l}-h_{0}\right)<\epsilon, \quad l=1,2, \ldots, M
$$

or, in a simpler form,

$$
\sup _{x \in \Omega}\left|R_{l}(s, x)-B_{l}(x)\right|<c \epsilon, \quad l=1,2, \ldots, M
$$

where

$$
\begin{gathered}
R_{l}=\sum_{i=1}^{N} M_{l i} s_{i}, \\
M_{l i}=a_{i} \sigma\left(b_{i} q_{l}-\bar{h}\right), \quad B_{l}=\sigma^{-1}\left(\lambda_{0} q_{l}-h_{0}\right) .
\end{gathered}
$$

Although $R_{l}$ are linear in $s_{i}$ functions, the left hand side of (68) is, in general, a nonlinear function of a complicated form. To overcome this difficulty, we replace the sup in (68) by the $L_{2^{-}}$norm that gives quadratic in $s$ functionals:

$$
\mathbf{R}_{l}(s)=\left\|R_{l}(s, x)-B_{l}(x)\right\|^{2}<c \epsilon^{2} . \quad l=1,2, \ldots, M
$$

We use Lagrange multipliers $\beta_{l}$ to take into account conditions (69). This leads to the following Lagrange function:

$$
\mathbf{F}(s)=\mathbf{H}(s)+\sum_{l=1}^{L} \beta_{l} \mathbf{R}_{l}(s)^{2}
$$

Let us remind that the matrix $W_{m m^{\prime}}$, that determines our hamiltonian $\mathbf{H}$, is a random matrix depending on random fields $\xi_{i}(x)$ through $\rho(\bar{\eta})$. Let us consider these fields as elements of the Banach $\operatorname{space} C^{0}(\Omega)$ of all bounded continuous in $x$ vector valued functions. Let $\mu_{\xi}$ be a probability measure defined on the subset of all such functions satisfying (58).

We also propose that variables $s$ are chosen by a stochastic algorithm. The stochastic algorithm depends on some set of parameters $P$ that can be adjusted. Let $\mu_{P}$ be a probability measure associated with this algorithm (this measure is defined below).

We would like to have a small value of $\mathbf{F}$ for a "most part" of field $\xi$ and $s$ values, with respect to the product measure $\mu=\mu_{\xi} \times \mu_{P}$.

Finally, the combinatorial problem can be formulated as follows: for a small number $\delta$, find parameters $P$ such that the probability (computed by the measure $\mu$ ),

$$
\operatorname{Prob}\{\mathbf{F}(\xi(\cdot), s)>\delta\}
$$

is small enough. 


\subsection{Mean field solution can be obtained by quadratic optimization}

We show here that the optimization problem is feasible when $N$ is large. To this end, we define the mean field Lagrange function $\overline{\mathbf{F}}$ that is obtained from $\mathbf{F}(\xi(\cdot), s)$ by averaging with respect to $\mu$. In order to estimate the deviations of $\mathbf{F}$ from $\overline{\mathbf{F}}$ we use the Chebyshev inequality:

$$
P(F, s)=\operatorname{Prob}\{|\mathbf{F}(\xi(\cdot), s)-\overline{\mathbf{F}}(s)|>a\} \leq a^{-2} \operatorname{Var} \mathbf{F},
$$

where the probability, the average and the variance should be computed by $\mu$.

The stochastic algorithm for choosing the satellites can be a simple Bernoulli scheme. Namely, let us consider $s_{i}$ as mutually independent random variables such that

$$
\operatorname{Prob}\left\{s_{i}=1\right\}=p_{i} .
$$

Thus, the mean field Lagrange function reads

$$
\overline{\mathbf{F}}(p)=\sum_{i=1}^{N} \sum_{j=1}^{N} \bar{W}_{i j} p_{i} p_{j}+\sum_{l=1}^{L} \beta_{l} \mathbf{R}_{l}(p)
$$

where $\bar{W}_{i j}$ is obtained from $W_{i j}$ by averaging with respect to $\mu_{\xi}$. Our main idea is as follows.

Step 1: Quadratic programming for the mean field Lagrange function

First, we minimize $\overline{\mathbf{F}}$ with respect to $p_{i}$. This is a quadratic programming problem that can be solved in polynomial time.

QP to find a minimum $\overline{\mathbf{F}}(p)$ under conditions

$$
\begin{gathered}
0 \leq p_{i} \leq 1, \\
R_{0}(p)=\sum_{i=1}^{N} p_{i}>0 .
\end{gathered}
$$

The last condition is trivial and can be omitted. Therefore, we look for a minimum of a positively defined quadratic form on the multidimensional box. The well-known L.Khachiyan ellipsoid algorithm for this problem runs in $\operatorname{Poly}(N)$ time. This proves such a lemma:

Lemma 4.5. If a solution of the problem $\mathbf{Q P}$ exists, then it can be found in Poly $(N)$ time.

Step 2: Obtain a small variance of the Lagrange function in the limit $N$ large

Let us suppose that $\overline{\mathbf{F}}<\delta / 2$. Then, using (72) we get

$$
\operatorname{Prob}\{\mathbf{F}(\xi(\cdot), s)>\delta\}<\operatorname{Prob}\{|\mathbf{F}(\xi(\cdot), s)-\overline{\mathbf{F}}(s)|>\delta / 2\} \leq 4 \delta^{-2} \operatorname{Var} \mathbf{F} .
$$

Now let us estimate $\operatorname{Var} \mathbf{F}$. We consider $\operatorname{Var} \mathbf{H}$, the rest terms $\mathbf{R}_{l}$ can be considered in a similar way. First we estimate variation with respect to $s$ by the measure $\mu_{P}$. One notices that

$$
\operatorname{Var} \mathbf{H}=E \sum_{i j i^{\prime} j^{\prime}} s_{i} s_{j} s_{i^{\prime}} s_{j^{\prime}} W_{i j} W_{i^{\prime} j^{\prime}}-E \sum_{i j} s_{i} s_{j} W_{i j} E \sum_{i^{\prime} j^{\prime}} s_{i^{\prime}} s_{j^{\prime}} W_{i^{\prime} j^{\prime}} .
$$

Notice that if $i \neq i^{\prime}$ and $j \neq j^{\prime}$ then

$$
E s_{i} s_{j} s_{i^{\prime}} s_{j^{\prime}}=E s_{i} s_{j} E s_{i^{\prime}} s_{j^{\prime}} .
$$

Moreover, $\left|W_{i j}\right|=O\left(N^{-2}\right)$ due to our assumption (55) on $a_{i}$ and Assumption 4.4. Thus we have maximum $N^{3}$ of non-zero terms in $D H$, which have the order $O\left(N^{-4}\right)$. Thus, the complete variation satisfies

$$
\operatorname{Var} \mathbf{H}<C_{0} N^{-1}
$$


where $C_{0}$ is uniform in $N$ as $N \rightarrow \infty$.

Thus, for large $N$ one has $\operatorname{Var} \mathbf{F} \rightarrow 0$, thus the probability (71) is arbitrarily small. This shows that the problem of minimization (72) is feasible in polynomial time $\operatorname{Poly}(N)$, when $N$ is large enough. More precisely, we have the following

Proposition 4.6. If a solution of the problem QP exists and $N$ is large enough, then a solution $s$ satisfying all restrictions and minimizing $F$ at level $\delta$ with a probability, arbitrarily close to 1 , can be found in Poly $(N)$ time.

Remark. Above we have studied the case $\xi_{0}=0$. For smooth $\xi_{0}(x)$ we can obtain a robustness with respect to $\xi_{0}$ variations in a simple way. Namely, for large $N$ one can choose the constant $C$ in (55)) large enough, then $\sum_{i=1}^{N} a_{i} u_{i}-h_{0}>>\left|\xi_{0}(x)\right|$.

There arises, however, a natural question: how genetic networks can realize these sophisticated algorithms which are capable to optimize the network robustness? A possible answer to this question is that $s_{i}$ could be themselves involved in a gene network of the form (11), (2). We showed that gene networks are capable to simulate all structurally stable dynamics. The fact that this is equivalent to simulating arbitrary Turing machines and thus arbitrary algorithms follows from results of Koiran and Moore (1999).

\section{Conclusion}

We are concerned with dynamical properties of networks with two types of nodes. The $v$-nodes, called centers, are hyperconnected and interact one to another via many $u$-nodes, called satellites. We show, by rigorous mathematical methods, that this centralized architecture, widespread in gene networks, allow to realize two fundamental biological strategies: flexible and robust bow-tie control and Wolpert positional information concepts.

We show how a combination of these strategies leads to the remarkable possibility to create a "multicellular organism", where each "cell" can exhibit a complicated time behaviour, different for different cells. Centralized network architectures provide the flexibility important in developmental processes and for adaptive functions.

Contrary to previous works on centralized boolean networks (Aldana 2003), we show that arbitrary bifurcations between attractors can be controlled by action on satellites, instead of actions on centers.

To check the robustness of such architectures we have considered a simplified example of a centralized network with a single center. Such system produces many equilibria, and this dynamical structure can be protected against large space dependent, random perturbations. We show that in general, designing an optimal network that is protected against such perturbations boils down to finding the minimum energy of a spin glass hamiltonian, which is a computationally hard problem. However, for a large number of satellites, the randomness is filtered and reliable protection against perturbations results as a solution to a quadratic programming problem, that can be solved in polynomial time. We expect that similar results hold more generally, for networks with any number of centers. This suggests an evolutionary bias towards centralized networks where hubs are subjected to control from many satellites.

These findings can be interpreted in terms of gene networks. The flexibility control by satellites, and not by transcription factors (centers) can be a major property of such networks. It may be easier to act on a satellite (by silencing or reactivating it), then to perform similar actions on a center (deletion of a hub proves most of the time to be lethal). We have proposed miRNAs and CREMs as possible candidates for satellite nodes in gene networks controlling pattering in development. A few examples of such centralized motifs are known, such for instance the enhancer system of the even-skipped gene of Drosophila (Ludwig et al 2011). The process of reconstruction of such networks is only at the beginning (see for instance (Berezikov 2011)). One could expect that many more examples of centralized motifs and networks will be found during this process. 
Acknowledgements. The authors are grateful to John Reinitz, Maria Samsonova and Vitaly Gursky for useful discussions. We are thankful to M. S. Gelfand and his colleagues for stimulating discussions in Moscow.

SV was supported by the Russian Foundation for Basic Research (Grant Nos. 10-01- 00627 s and 10-01-00814 a) and the CDRF NIH (Grant No. RR07801) and by a visiting professorship grant from the University of Montpellier 2.

\section{Appendix: Proofs and estimates}

\section{The proof of Proposition 2.1}

To outline the proof, let us notice that our system has a typical form, where slow $(v)$ and fast $(u)$ components are separated:

$$
v_{t}=\kappa F(v, u), \quad u_{t}=A u+\kappa G(v) .
$$

Let us present $u$ as $u=U+\tilde{u}$, where $U=-\kappa A^{-1} G(v)$ and $\tilde{u}$ is a new unknown. Let us notice that $U_{i}$ are solutions of (18) under boundary conditions (5) and that $\left|U_{i}\right|<c \kappa$.

By substituting $u=U+\tilde{u}$ into (78), we obtain

$$
v_{t}=\kappa F(v, U+\tilde{u}), \quad \tilde{u}_{t}=A \tilde{u}+\kappa^{2} G_{1}(v, U+\tilde{u}),
$$

where $G_{1}=\kappa^{-1} A^{-1} G^{\prime}(v) v_{t}=A^{-1} F(v, U+\tilde{u})$, the operator $A=\operatorname{diag}\left\{\tilde{d}_{i} \Delta-\tilde{\lambda}_{i}\right\}$. Let us show that $G_{1}(\tilde{u}, v)$ is a uniformly bounded map in $\mathcal{H}$ for all $u, v$ satisfying a priori estimates (10). For sufficiently smooth initial data $\phi, \tilde{\phi} \in C^{2}$ these estimates and evolution equation (9) imply

$$
\|v(t)\|_{\alpha} \leq C_{1}, \quad t \geq 0, \alpha \in(0,1) .
$$

The Sobolev embedding gives then

$$
\|\nabla v(t)\|_{L_{4}(\Omega)} \leq c\|v(t)\|_{\alpha} \leq C_{2}, \quad t \geq 0, \alpha \in(1 / 2,1) .
$$

To estimate now $G_{1}=\left(w_{1}, \ldots, w_{N}\right)^{t r}$, let us notice that $w_{i}$ satisfy the following equations:

$$
\left(\tilde{d}_{i} \Delta-\tilde{\lambda}_{i}\right) w_{i}=g_{i}(x, v)\left(d_{i} \Delta-\lambda_{i}\right) v_{i},
$$

where $g_{i}$ are smooth functions with uniformly bounded derivatives. Our goal is, thus, to estimate $\|\nabla w\|$ through $\|\nabla v\|_{L_{4}}$ and $\|v\|_{\alpha}$. Let us multiply (82) through $w_{i}$ and then integrate the left hand and the right hand sides of the obtained equations by parts. We find

$$
\left\|\nabla w_{i}\right\|^{2} \leq c_{1}|| \nabla w_{i}\left|\left\||| \nabla v_{i}\right\|+c_{2}\left\langle(\nabla v)^{2},|w|\right\rangle\right. \text {. }
$$

To estimate $\left\langle(\nabla v)^{2},|w|\right\rangle$, we use the Cauchy-Schwartz inequality

$$
\left|\left\langle(\nabla v)^{2},|w|\right\rangle\right| \leq c|| \nabla v\left\|_{L_{4}(\Omega)}\right\| w \|,
$$

Now we can apply (81) and the Cauchy inequality with a parameter $a>0$ that gives

$$
\|\nabla w\|\|\nabla v\| \leq c_{1} a\|\nabla w\|^{2}+C a^{-1}\|\nabla v\|^{2},
$$

and if $a>0$ is small enough $\left(c_{1} a<1\right)$, we obtain, by (83) and (84), the need estimate:

$$
\|\nabla w\|<C_{3} .
$$

The second equation in (79) then entails

$$
\|\tilde{u}\|_{t} \leq-\beta\|\tilde{u}\|+\kappa^{2} \sup \left\|G_{1}\right\|,
$$


where $\beta=\min \left\{\tilde{\lambda}_{i}\right\}>0$ is independent of $\kappa$. This gives

$$
\|\tilde{u}(t)\| \leq\|\tilde{u}(0)\| \exp (-\beta t)+C_{4} \kappa^{2} .
$$

In a similar way one can obtain the same estimate for $\|\nabla \tilde{u}\|$. This completes the proof.

\section{Estimates for network viability via spin hamiltonian}

Assume that for some $\xi(x)=\left(\xi_{1}(x), \ldots, \xi_{N}(x)\right)$ there holds

$$
\mathbf{H}(x, \xi(\cdot))<\delta .
$$

Let us obtain estimates of deviations $\tilde{v}=v-q$ and $\tilde{u}_{i}=u_{i}-U_{i}(q)$, where $v=q(x), u=U_{i}$ define an equilibrium stationary solution for $\xi_{i}(x) \equiv 0$. These estimates hold only due to the special structure of our network: we admit that $\xi_{i}$ are not small, nonetheless, the summarized effect of these perturbations is small. We assume

$$
\tilde{u}_{i}(x, 0)=0, \quad \tilde{v}(x, 0)=0 .
$$

Let us present the functions $\tilde{u}_{i}$ as sums $\tilde{u}_{i}=\bar{\eta}_{i}+w_{i}$, where $\bar{\eta}_{i}$ are defined by (61). For $w_{i}, \tilde{v}$ we then obtain

$$
\begin{gathered}
\frac{\partial \tilde{v}}{\partial t}=d_{0} \Delta \tilde{v}-\lambda_{0} v+\sigma(\rho(U+\tilde{u}(\tau))-\bar{h})-\sigma(\rho(U)-\bar{h}), \\
\frac{\partial w_{i}(t)}{\partial t}=d_{i} \Delta \tilde{v}-\lambda_{i} v+F_{i}(\tilde{v}(\tau), \xi) d \tau
\end{gathered}
$$

where

$$
F_{i}(\tilde{v}, \xi)=\sigma\left(b_{i}(q+\tilde{v})-h_{i}+\xi_{i}\right)-\sigma\left(b_{i} q-h_{i}+\xi_{i}\right) .
$$

Let us observe that

$$
\left\|\tilde{F}_{i}\right\|<c\|\tilde{v}\|
$$

Condition (85) implies that

$$
\|\rho(\eta(\cdot))\|<\delta .
$$

Let us introduce $\|w\|$, by $\|w\|^{2}=\sum_{i=1}^{N}\left\|w_{i}\right\|^{2}$ and $|a|$ by $|a|^{2}=\sum_{i=1}^{N}\left|a_{i}\right|^{2}$. Then

$$
\|\rho(w)\| \leq|a||| w|| .
$$

By (87), (88), (89) and (90) now one obtains inequalities for $\|\tilde{v}\|,\left\|w_{i}\right\|$ :

$$
\begin{gathered}
\frac{d\|\tilde{v}\|^{2}}{2 d t} \leq-\lambda_{0}\|\tilde{v}\|^{2}+c_{3}(\|\rho(\bar{\eta})\|+|a|\|w\|), \\
\frac{d\|w\|^{2}}{2 d t} \leq-\bar{\lambda}\|w\|^{2}+c_{4}\|\tilde{v}\| .
\end{gathered}
$$

where $\min _{i} \lambda_{i}=\bar{\lambda}>0$. Assume that $\min _{i} \lambda_{i}>0$ are large enough. Moreover, for large $N$ the coefficient $c_{3}|a|<1$. Combining (91), (92) one obtains the inequality for $\|Y\|^{2}=\|w\|^{2}+\|\tilde{v}\|^{2}$ :

$$
\frac{d\|Y\|^{2}}{2 d t} \leq-\lambda_{0}\|Y\|^{2}+\left(\lambda_{0}-\bar{\lambda}\right)\|w\|^{2}+c_{3} \delta\|\tilde{v}\|+c_{5}\|w\|\|\tilde{v}\| .
$$

We apply now the Cauchy inequality $x y<a x^{2}+a^{-1} y^{2}$ to the two terms in the right hand side of this last inequality. This gives

$$
\frac{d\|Y\|^{2}}{2 d t} \leq-\lambda_{0}\|Y\|^{2}+\left(\lambda_{0}-\bar{\lambda}\right)\|w\|^{2}+a^{-1}\|w\|^{2}+c_{6} a\|\tilde{v}\|^{2}+c_{7} a^{-1} \delta^{2}\|\tilde{v}\| .
$$


We adjust an $a$ such that $c_{0} a<\lambda_{0} / 2$. If $\bar{\lambda}$ is large enough, 94 gives then

$$
\|Y(t)\| \leq c \delta+\|Y(0)\| \exp \left(-\lambda_{0} t / 2\right) .
$$

Then (95) implies that for large $t$ there holds

$$
\sup _{t>0}\|\tilde{v}(t)\|_{\alpha} \leq c_{9} \delta
$$

with a constant $c_{9}>0$. This gives us the need estimate of $\tilde{v}$ via the spin hamiltonian.

\section{References}

[1] M. Aldana, Boolean dynamics of networks with scale-free topology, Physica D 185, 45 - 66, 2003.

[2] M. Aldana, and P. Cluzel, A natural class of robust networks, Proc. Natl. Acad. Sci. U.S.A. 100, $8710-8714,2003$.

[3] R. Albert and A.L. Barabási, Rev. Modern Physics 74, 47-97, 2002.

[4] D.V. Anosov (ed), Dynamical Systems 9: Dynamical Systems with Hyperbolic Behaviour, Encyclopedia of Mathematical Sciences Vol. 66. Translated from Russian., Springer V., Berlin, Heidelberg, New-York, 1995.

[5] J.P. Aubin, A. Bayen, N. Bonneuil and P. Saint-Pierre, Viability, Control and Games: Regulation of complex evolutionary systems under uncertainty and viability constraints, Springer-Verlag. 2005

[6] A.B. Babin and M.I. Vishik, Regular attractors of semigroups and evolution equations, J. Math. Pure Appl. 62, 441 - 491, 1983.

[7] A. Barron, Universal Approximation Bounds for superpositions of a sigmoidal functions, IEEE Trans. on Inf. theory 39, 930-945. 1993

[8] E. Berezikov, Evolution of microRNA diversity and regulation in animals Nature Reviews Genetics 12, 846-860, 2011.

[9] S.N. Chow and K. Lu, Invariant manifolds for flows in Banach spaces J. Differential equations 74, 285-317, 1988.

[10] P. Constantin, C. Foias, B. Nicolaenko and R. Temam, Integrable manifolds and inertial manifolds for dissipative differential equations, Springer, New-York, 1989.

[11] M. Csete and J. Doyle, Bow ties, metabolism and disease. Trends Biotechnol 22, 446-450, 2004.

[12] E.N. Dancer and P. Poláčik, Realization of vector fields and dynamics of spatially homogeneous parabolic equations. Memoirs of Amer. Math. Society 140, no. 668, 1999.

[13] M. Delbrück, Discussion: Unitées biologiques douées de continuité génétique, Actes du colloque international du CNRS, pp. 33-3, Editions du CNRS, Paris, 1949.

[14] K. Funahashi and Y. Nakamura, Approximation of dynamical systems by continuous time recurrent neural networks. Neural Networks 6, 801-806, 1993.

[15] D. Henry, Geometric Theory of Semiliniar Parabolic Equations. Springer, New York, 1981. 
[16] J.J. Hopfield, Neural networks and physical systems with emergent collective computational abilities, Proc. of Natl. Acad. USA 79, 2554-2558, 1982.

[17] S.A. Kauffman, Metabolic stability and epigenesis in randomly constructed genetic nets. J. Theor. Biol. 22, 437-67, 1969 .

[18] P. Koiran and C. Moore, Closed-form analytic maps in one and two dimensions can simulate universal Turing machines. Theoretical Computer Science 210, 217-223, 1999.

[19] A. Lesne, Complex networks: from graph theory to biology. Letters in Math. Phys. 78, 235-262, 2006.

[20] X. Li, J.J. Cassidy, C.A. Reinke, S. Fischboeck, and R.W. Carthew, A MicroRNA Imparts Robustness against Environmental Fluctuation during Development, Cell 137, 273-282, 2009.

[21] M.Z. Ludwig, Manu, P. Kittler, K.P. White, and M. Kreitman, Consequences of Eukaryotic Enhancer Architecture for Gene Expression Dynamics, Development, and Fitness, PLoS Genetics 7, e1002364, 2011.

[22] H. Ma, A. Sorokin, A. Mazein, A. Selkov, E. Selkov, O. Demin, and I. Goryanin, The Edinburgh human metabolic network reconstruction and its functional analysis, Molecular Systems Biology 3, 135, 2007.

[23] R. Mane, Reduction of semilinear parabolic equations to finite dimensional $C^{1}$ - flow, Geometry and Topology, Lecture Notes in Mathematics, No. 597, Springer -Verlag, New -York, 361-378, 1977.

[24] M. Marion, Approximate inertial manifolds for reaction-diffusion equations in high space dimension, J. Dyn. Diff. Equations 1, 245-267, 1989.

[25] N.J. Martinez, M.C. Ow, M.I. Barrasa, M. Hammell, R. Sequerra, L. Doucette-Stamm, F.P. Roth, V.R. Ambros, and A.J.M. Walhout, A C. elegans genome-scale microRNA network contains composite feedback motifs with high flux capacity, Genes Dev. 22, 2535-2549, 2008.

[26] H. Meinhardt, Models of biological pattern formation, Academic Press, London, 1982.

[27] M. Mezard and R. Zecchina, Random k-satisfiability problem: From an analytic solution to an efficient algorithm, Phys. Rev. E 66, 056126, 2002.

[28] E. Mjolness, D.H. Sharp and J. Reinitz, A connectionist model of development, J. Theor. Biol. 152, 429-453, 1991.

[29] J.D. Murray, Mathematical Biology, Springer, New York, 1993.

[30] M.H.A. Newman, Alan Mathison Turing. 1912-1954, Biogr.Mems Fell.R.Soc. 1, 253-263, 1955.

[31] R. Newhouse, D. Ruelle and F. Takens, Occurence of strange axiom A attractors from quasi periodic flows, Comm.Math. Phys. 64, 35-40, 1971.

[32] K.M. Page, P.K. Maini and N.A.M. Monk, Complex pattern formation in reaction-diffusion systems with spatially varying parameters. Physica D 202, 95-115, 2005.

[33] M.E. Peter, Targeting of mRNAs by multiple miRNAs: the next step. Oncogene 29, 2161-2164, 2010.

[34] P. Poláčik, Complicated dynamics in Scalar Semilinear Parabolic Equations, In Higher Space Dimensions Journ. of Diff. Eq. 89, $244-271,1991$. 
[35] M.I. Rabinovich and H.D.I. Abarbanel, The role of chaos in neural systems, Neuroscience 87 (N1), 5-14, 1998.

[36] J. Reinitz and D. H. Sharp, Mechanism of formation of eve stripes, Mechanisms of Development 49, 133-158, 1995.

[37] D. Ruelle, Elements of differentiable dynamics and bifurcation theory. Acad. Press, Boston, 1989.

[38] D. Ruelle and F. Takens, On the nature of turbulence, Comm. Math. Phys 20, 167 -192, 1971.

[39] K.P. Rybakowski, Realization of arbitrary vector fields on center manifolds of parabolic Dirichlet BVP's, J. Differential Equations 114, 199-221, 1994.

[40] M. Scott, F.J. Poulin and H. Tang, Approximating intrinsic noise in continuous multispecies models. Proc. Roy. Soc. A 467, 718-737, 2011.

[41] R. Shalgi, D. Lieber, M. Oren, Y. Pilpel, Global and Local Architecture of the Mammalian microRNATranscription Factor Regulatory Network. Plos Comp. Bio. 3, 1291-1304, 2007.

[42] H.T. Siegelmann and E.D. Sontag, Turing computability with neural networks. Appl. Math. Lett. 4, 6, 1991.

[43] H.T. Siegelmann and E.D. Sontag, On the computational power of neural nets. J. Comp. Syst. Sci. 50, 132-150, 1995.

[44] S. Smale, Mathematics of Time, Springer, New - York. 1980.

[45] M. Talagrand, Spin glasses, a Challenge for Mathematicians. Springer-Verlag. 2003.

[46] K.C. Tu and B.L. Bassler, Multiple small RNAs act additively to integrate sensory information and control quorum sensing in Vibrio harveyi. Genes Dev. 21, 221-233, 2007.

[47] C. Teuscher and E. Sanchez, A revival of Turings forgotten connectionist ideas: exploring unorganized machines. Springer-Verlag, London, 2001.

[48] R. Thomas, Laws for the dynamics of regulatory networks. Int J Dev Biol. 42, 479485, 1998.

[49] A.M. Turing, The chemical basis of morphogenesis. Phil. Trans. Roy. Soc. B 237, 37-72, 1952.

[50] A.M. Turing, Intelligent Machinery. In B. Melzer and D. Michie, editors, Machine Intelligence, volume 5 of National Physical Laboratory Report, 3-23, Edinburgh University Press, Edinburgh, 1969.

[51] S.A. Vakulenko, A system of coupled oscillators can have arbitrary prescribed attractors. J. Phys. A. Math. Gen. 27, 2335-2349, 1994.

[52] S.A. Vakulenko and P.V. Gordon, Neural networks with prescribed large time behaviour. J. Phys. A. Math. Gen 31, 9555-9570, 1998.

[53] S.A. Vakulenko, Dissipative systems generating any structurally stable chaos, Advances in Diff. Equations 5, 1139-1178, 2000.

[54] L. Wolpert, Positional information and pattern formation. In C.H. Waddington, editor, Towards a theoretical biology 3.Drafts. Aldine Publishing Company, Chicago, 1970.

[55] L. Wolpert, R. Beddington, T. Jessell, P. Lawrence, E. Meyerowitz and J. Smith, Principles of Development. Oxford University Press, Oxford, 2002. 
[56] S. Wu, S. Huang, J. Ding, Y. Zhao, L. Liang, T. Liu, R. Zhan and X. He, Multiple microRNAs modulate p21Cip1/Waf1 expression by directly targeting its 3' untranslated region. Oncogene 29, $2302-2308,2010$. 